\title{
NEW EXISTENCE OF EQUILIBRIA VIA CONNECTEDNESS
}

\author{
SUNG MO IM and DONG IL RIM \\ Department of Mathematics \\ Chungbuk National University \\ Cheongju 361-763, KOREA \\ and \\ WON KYU KIM \\ vepartment of Mathematics Education \\ Chungbuk National University \\ Cheongju 361-763, KOREA
}

(Received April 23, 1996 and in revised form September 4, 1996)

\begin{abstract}
The purpose of this paper is to prove a new topological fixed point theorem by using the connectedness property, and next existence of equilibria in generalized games are established as applications.
\end{abstract}

KEY WORDS AND PHRASES: Connectedness, closed multimap, upper semicontinuous, fixed point, equilibrium. .

1991 AMS SUBJECT CLASSIFICATION CODES: 47N10, 54C60, 54D05, 90A14.

\section{INTRODUCTION}

In many problems of nonlinear functional analysis and applied mathematics, the fixed point technique is a very useful tool for proving the existence of solutions. And we have known many interesting fixed point theorems and their applications, e.g. see Browder [1], Fan [2], Mehta and Tarafdar [3], Tan and Yuan [4].

The classical fixed point theorem, due to Fan and Browder [1,2], is as follows.

Let $X$ be a non-empty compact convex subset of a Hausdorff topological vector space $E$ and let $T: X \rightarrow 2^{X}$ be a multimap such that for each $x \in X, T(x)$ is non-empty convex and for each $y \in X, T^{-1}(y)$ is open in $X$. Then there exists $\bar{x} \in X$ such that $\bar{x} \in T(\bar{x})$.

In the above, the convexity assumption is very essential and the main proving tool is the continuous selection technique. Still there have been many equivalent formulations of the above theorem, and also many generalizations and applications have been established, e.g. see $[3,4,5]$.

In this paper, we shall give a new existence theorem by using the topological property of convex sets We first prove a new fixed point theorem by using the connectedness, and next prove a basic existence theorem. Finally equilibrium existence theorems are established as applications.

\section{PRELIMINARIES}

We first recall the following notations and definitions. Let $A$ be a non-empty set. We shall denote by $2^{A}$ the family of all subsets of $A$. Let $X, Y$ be non-empty topological spaces and $T: X \rightarrow 2^{Y}$ be a multimap. The multimap $T$ is said to be open or have open graph if the graph of ' $T$ ' ( $G T=\{(x, T(x)) \in X \times Y \mid x \in X\}$ ) is open in $X \times Y$, and $T$ is said to be closed or have closed graph if the graph of $T$ is closed in $X \times Y$. We may call $T(x)$ the upper section of $T$, and $T^{-1}(y)(=\{x \in X \mid y \in T(x)\})$ the lower section of $T$. It is easy to check that if $T$ has open graph, then the upper and lower sections of $T$ are open; however the converse is not true in general. 
A multimap $T: X \rightarrow 2^{Y}$ is said to be closed at $x$ if for each net $\left(x_{\alpha}\right) \rightarrow x, y_{\alpha} \in T\left(x_{\alpha}\right)$ and $\left(y_{\alpha}\right) \rightarrow y$, then $y \in T(x)$. A multimap $T$ is closed if it is closed at every point of $X$. It is clear that if $T$ is closed at $x$ and each $T\left(x_{\alpha}\right)$ is non-empty, then $T(x)$ is also non-empty. Note that if $T$ is single-valued, then the closedness is equivalent to continuity as a function. Let $S, T: X \rightarrow 2^{Y}$ be multimaps; then $S$ is called a closed selection of $T$ if for each $x \in X, S(x) \subset T(x)$ and $S$ is a closed multimap. Let $X, Y$ be non-empty topological spaces and $T: X \rightarrow 2^{Y}$ be a correspondence. A correspondence $T: X \rightarrow 2^{Y}$ is said to be upper semicontimuous if for each $x \in X$ and each open set $V$ in $Y$ with $T(x) \subset V$, then there exists an open neighborhood $U$ of $x$ in $X$ such that $T(y) \subset V$ for each $y \in U$.

For a given multimap $T: X \rightarrow 2^{Y}, x \in X$ is called a maximal element for $T$ if $T(x)=\emptyset$. Indeed, in real applications, the maximal element may be interpreted as the set of those objects in $X$ that are the "best" or "largest" choices.

Finally we recall the following definitions of equilibrium theory in mathematical economics. Let $I$ be a finite or an infinite set of agents. For each $i \in I$, let $X_{i}$ be a non-empty set of actions A generalized game $\Gamma=\left(X_{2}, A_{2}, P_{2}\right)_{2 \in I}$ is defined as a family of ordered triples $\left(X_{2}, A_{2}, P_{2}\right)$ where $X_{2}$ is a non-empty topological space (a choice set), $A_{2}: \Pi_{\jmath \in I} X_{\jmath} \rightarrow 2^{X_{1}}$ is a constraint correspondence (multimap) and $P_{\imath}: \Pi_{\jmath \in I} X_{j} \rightarrow 2^{X_{i}}$ is a preference correspondence. An equilibrium for $\Gamma$ is a point $\widehat{x} \in X=\Pi_{\imath \in I} X_{\imath}$ such that for each $i \in I, \widehat{x}_{i} \in A_{i}(\widehat{x})$ and $A_{i}(\widehat{x}) \cap P_{\imath}(\widehat{x})=\emptyset$. In particular, when $I=\{1, \cdots, n\}$, we may call $\Gamma$ an $N$-person game.

\section{A NEW FIXED POINT THEOREM}

We begin with the following:

THEOREM 1. Let $X$ be a non-empty connected subset of a Hausdorff topological space $E$ and $S, T: X \rightarrow 2^{E}$ be multimaps such that

(1) $S$ is a closed selection of $T$, i.e. $S$ is a closed multimap and $S(x) \subset T(x)$ for each $x \in X$,

(2) for some $y_{0} \in E, S^{-1}\left(y_{0}\right)$ is non-empty open in $X$.

Then we have

$$
\left\{y_{0}\right\} \subset \bigcap_{x \in X} T(x) .
$$

In particular, if $y_{0} \in X$, then $y_{0}$ is a fixed point for $T$, i.e. $y_{0} \in T\left(y_{0}\right)$.

PROOF. Since $S$ is closed, the lower section $S^{-1}\left(y_{0}\right)$ is closed. In fact, for every net $\left(x_{\alpha}\right)_{\alpha \in \Gamma} \subset S^{-1}\left(y_{0}\right)$ with $\left(x_{\alpha}\right) \rightarrow x$, we have $y_{0} \in S\left(x_{\alpha}\right)$ for each $\alpha \in \Gamma$ and $\left(x_{\alpha}\right) \rightarrow x$, so by the closedness of $S$ at $x, y_{0} \in S(x)$. Hence $S(x) \neq \emptyset$ and $x \in S^{-1}\left(y_{0}\right)$, so that $S^{-1}\left(y_{0}\right)$ is closed. By assumption (2), $S^{-1}\left(y_{0}\right)$ is both open and closed in $X$, and hence by the connectedness of $X, S^{-1}\left(y_{0}\right)=X$. Therefore we have $y_{0} \in S(x) \subset T(x)$ for each $x \in X$, so that $\left\{y_{0}\right\} \subset \cap_{x \in X} T(x)$. In particular, if $y_{0} \in X$, then we have $y_{0} \in T\left(y_{0}\right)$. This completes the proof.

We can reformulate Theorem 1 to the following existence of maximal element:

COROLLARY. Let $X$ be a non-empty connected subset of a Hausdorff topological space $E$ and $T: X \rightarrow 2^{X}$ be closed at every $x$ where $T(x) \neq \emptyset$, such that

(1) $T^{-1}\left(y_{0}\right)$ is non-empty open in $X$ for some $y_{0} \in X$,

(2) $y_{0} \notin T\left(y_{0}\right)$.

Then $T$ has a maximal element $\bar{x} \in X$, i.e. $T(\bar{x})=\emptyset$.

PROOF. Suppose $T(x) \neq \emptyset$ for each $x \in X$. Then by Theorem $1, y_{0} \in T(x)$ for each $x \in X$, and this contradicts assumption (2). Therefore $T$ has a maximal element.

As we remarked before, maximal element theorem is a very essential tool for proving existence theorems in mathematical economics, e.g. see $[3,4,5]$.

In Theorem 1, if we assume the strong open lower section condition on $T$, we can obtain the following: 
THEOREM 2. Let $X$ be a non-empty connected subset of a Hausdorff topological space $E$ and $T: X \rightarrow 2^{E}$ be a closed multimap such that

(1) for some $x_{0} \in X, T\left(x_{0}\right)$ is non-empty,

(2) for each $y \in E, T^{-1}(y)$ is open (maybe empty) in $X$.

Then there exists a non-empty subset $K \subset E$ such that $T(x)=K$ for each $x \in X$, i.e. $T$ is a constant multimap.

PROOF. Suppose the assertion were false. Then we can find $x_{1} \in X$ and $y_{0} \in E$ such that either $y_{0} \in T\left(x_{0}\right) \backslash T\left(x_{1}\right)$ or $y_{0} \in T\left(x_{1}\right) \backslash T\left(x_{0}\right)$. Suppose the case that $y_{0} \in T\left(x_{0}\right) \backslash T\left(x_{1}\right)$. Since $T$ is closed, as in the proof of Theorem 1, the lower section $T^{-1}\left(y_{0}\right)$ is closed. By assumption (2), $T^{-1}\left(y_{0}\right)$ is also open Since $x_{0} \in T^{-1}\left(y_{0}\right)$, by the connectedness of $X, T^{-1}\left(y_{0}\right)=X$. Therefore we have $y_{0} \in T(x)$ for each $x \in X$, which is a contradiction. In the case of $y_{0} \in T\left(x_{1}\right) \backslash T\left(x_{0}\right)$, we can also show that $T^{-1}\left(y_{0}\right)$ is both open and closed, so that this induces the same contradiction. Therefore $T$ must be constant, i.e. there exists a non-empty subset $K \subset E$ such that $T(x)=K$ for each $x \in X$. This completes the proof.

The above theorem can be useful in the following examples:

EXAMPLES. (i) Let $X:=(0,1]$ and $S, T: X \rightarrow 2^{X}$ be defined as follows:

$$
\begin{aligned}
& T(x)=\left\{\begin{array}{lll}
\left(\frac{1}{2} x+\frac{1}{2}, 1\right], & \text { if } 0<x \leq \frac{1}{2}, \\
{\left[\frac{1}{4} x+\frac{3}{4}, 1\right],} & \text { if } \frac{1}{2}<x \leq 1 .
\end{array}\right. \\
& S(x):=\left[\frac{1}{4} x+\frac{3}{4}, 1\right], \quad \text { for each } x \in X .
\end{aligned}
$$

Then it is easy to see that $S$ is clearly a closed selection of $T$ and $S$ satisfies the whole assumptions of Theorem 1. Therefore $T$ has a fixed point. Note that since $S^{-1}(y)$ is not necessarily open, we cannot apply Theorem 2 directly to $S$.

(ii) Let $X:=\left\{(x, y) \in R^{2}: y=\sin \frac{1}{x}, x>0\right\} \cup\{(0,0)\}$ be the topological sine curve in $R^{2}$ and a multimap $T: X \rightarrow 2^{X}$ be defined as follows:

$$
T(x, y)=\left\{\begin{array}{ccc}
X, & \text { if } & (x, y)=(0,0) \\
\{(0,0)\}, & \text { if } & (x, y) \neq(0,0)
\end{array}\right.
$$

Then $T$ is clearly closed at every point in the connected set $X$ and also $T^{-1}(0,0)=X$ is open. Therefore, by Theorem 1, we can find a fixed point for $T$. And it should be noted that the domain of $T$ and some $T(x)$ are not convex, so that many known fixed point theorems (e.g. $[1,2,3,5,6]$ cannot be suitable for $T$.

\section{EXISTENCE OF EQUILIBRIA}

As an application, we first give an existence theorem of equilibrium for 1-person game

THEOREM 3. Let $\Gamma=(X, A, P)$ be a 1-person game such that

(1) $X$ is a non-empty connected subset of a Hausdorff topological space,

(2) the correspondence $A: X \rightarrow 2^{X}$ is closed such that $A(x)$ is connected for some $x \in X$,

(3) the correspondence $P: X \rightarrow 2^{X}$ is closed at every $x$ where $A(x) \cap P(x) \neq \emptyset$,

(4) for each $y \in X, A^{-1}(y)$ is open (maybe empty) in $X$,

(5) for some $y_{0} \in X, A^{-1}\left(y_{0}\right) \cap P^{-1}\left(y_{0}\right)$ is non-empty open in $X$,

(6) $y_{0} \notin P\left(y_{0}\right)$.

Then $\Gamma$ has an equilibrium choice $\widehat{x} \in X$, i.e

$$
\widehat{x} \in A(\widehat{x}) \text { and } A(\widehat{x}) \cap P(\widehat{x})=\emptyset .
$$

PROOF. By assumptions (5), $A^{-1}\left(y_{0}\right)$ is non-empty and so $A\left(x_{0}\right)$ is non-empty for some $x_{0} \in A^{-1}\left(y_{0}\right)$. By assumptions (2) and (4), using Theorem 2, there exists a non-empty subset $K \subset X$ 
such that $A(x)=K$ for each $x \in X$. Then by assumption (2) again, $K$ is connected and the set $\{x \in X: x \in A(x)\}$ is exactly equal to $K$. We now consider a multimap $A \cap P: K \rightarrow 2^{K}$ defined by

$$
(A \cap P)(x):=A(x) \cap P(x) \text { for each } x \in K .
$$

In fact, $(A \cap P)(x)=P(x) \cap K$ for each $x \in K$. Then by assumptions (2) and (3), $A \cap P$ is closed at every $x$ where $A(x) \cap P(x) \neq \emptyset$. Therefore the whole assumptions of the Corollary are satisfied, so that there exists some $\widehat{x} \in K$ such that $A(\widehat{x}) \cap P(\widehat{x})=\emptyset$. Therefore $\widehat{x}$ is the desired equilibrium choice for $\Gamma$.

In the above, when $A(x)=X$ for each $x \in X$, we can obtain the previous Corollary as an application. And, by modifying the method in [4,5], we can generalize Theorem 3 to $\mathrm{N}$-person game or generalized games with infinitely many agents.

We shall need the following result which can be found in Aubin and Cellina [7]:

LEMMA. Let $X$ and $Y$ be regular topological spaces and $T: X \rightarrow 2^{Y}$ be an upper semicontinuous multimap such that for each $x \in X, T(x)$ is non-empty closed. Then $T$ is a closed multimap.

Finally, using different assumptions from Theorem 3, we can obtain the following existence of equilibrium for $\mathrm{N}$-person game:

THEOREM 4. Let $\Gamma=\left(X_{2}, A_{\imath}, P_{\imath}\right)_{\imath \in I}$ be a generalized game where $I=\{1, \cdots, n\}$ is a finite set of agents such that for each $i \in I$,

(1) $X_{2}$ is a non-empty connected subset of a normal topological space,

(2) the correspondence $A_{2}: X=\Pi_{\jmath \in I} X_{\jmath} \rightarrow 2^{X_{2}}$ is upper semicontinuous such that for each $x \in X, A_{2}(x)$ is a non-empty closed subset of $X_{2}$,

(3) the correspondence $P_{2}: X \rightarrow 2^{X_{2}}$ is upper semicontinuous such that for each $x \in X, P_{i}(x)$ is a closed subset of $X_{i}$,

(4) there exists some $y_{2} \in X_{i}$ such that $A_{i}^{-1}\left(y_{i}\right)$ and $(A \cap P)_{i}^{-1}\left(y_{i}\right)$ are non-empty open in $X$,

(5) the set $W_{2}:=\left\{x \in X:\left(A_{2} \cap P_{2}\right)(x) \neq \emptyset\right\}$ is (possibly empty) closed,

(6) for each $x \in W_{2}, x_{2} \notin P_{2}(x)$.

Then $\Gamma$ has an equilibrium choice $\widehat{y} \in X$, i.e. for each $i \in I, \widehat{y}_{2} \in A_{2}(\widehat{y})$ and $A_{2}(\widehat{y}) \cap P_{2}(\widehat{y})=\emptyset$

PROOF. Since each $X_{i}$ is connected, $X=\Pi_{2 \in I} X_{i}$ is also connected. And note that the correspondence $A:=\Pi_{i \in I} A_{\imath}: X \rightarrow 2^{X}$, defined by $A(x)=\prod_{i \in I} A_{\imath}(x)$ for each $x \in X$, is upper semicontinuous and each $A(x)$ is closed. Therefore, by the Lemma, $A$ is a closed multimap.

If $W_{\imath}=\emptyset$ for all $i \in I$, then by applying Theorem 1 to the correspondence $A: X \rightarrow 2^{X}$, there exists a point $\widehat{y}=\left(y_{1}, \cdots, y_{n}\right) \in X$ such that $\widehat{y} \in A(\widehat{y})$, i.e. for each $i \in I, y_{i} \in A_{i}(\widehat{y})$ and $A_{i}(\widehat{y}) \cap P_{i}(\widehat{y})=\emptyset$ Therefore, we are done.

Suppose that $I_{0}$ is a maximal non-empty subset of $I$ such that $W_{\imath} \neq \emptyset$ for all $i \in I_{0}$. Note that by assumptions (2) and (3), we can obtain the fact that the correspondence $A_{\imath} \cap P_{\imath}$ is also upper semicontinuous on $W_{i}$ for every $i \in I_{0}$.

For each $i \in I_{0}$, we now define a correspondence $\phi_{2}: X \rightarrow 2^{X_{2}}$ by

$$
\phi_{\imath}(x)= \begin{cases}A_{i}(x), & \text { if } x \notin W_{i}, \\ \left(A_{\imath} \cap P_{\imath}\right)(x), & \text { if } x \in W_{\imath} .\end{cases}
$$

Then for each $x \in X, \phi_{1}(x)$ is a non-empty closed subset of $X_{2}$. To show $\phi_{2}$ is upper semicontinuous, we must show that the set $U:=\left\{x \in X: \phi_{2}(x) \subset V\right\}$ is open in $X$ for every open subset $V$ of $X_{2}$. Now we have

$$
\begin{aligned}
U & =\left\{x \in W_{2}: \phi_{i}(x) \subset V\right\} \cup\left\{x \in X \backslash W_{2}: \phi_{1}(x) \subset V\right\} \\
& =\left\{x \in W_{2}:\left(A_{2} \cap P_{2}\right)(x) \subset V\right\} \cup\left\{x \in X \backslash W_{2}: A_{2}(x) \subset V\right\} \\
& =\left\{x \in X:\left(A_{\imath} \cap P_{2}\right)(x) \subset V\right\} \cup\left\{x \in X \backslash W_{i}: A_{2}(x) \subset V\right\}
\end{aligned}
$$


Since $X \backslash W_{2}$ is open and $A_{2} \cap P_{2}$ is upper semicontinuous, $U$ is open and hence $\phi_{i}$ is upper semicontinuous. Therefore, by the Lemma, $\phi_{2}$ is a closed multimap.

Next we shall show that $\phi_{\imath}^{-1}\left(y_{2}\right)$ is an open subset of $X$. In fact, by assumptions (4) and (5), we have that

$$
\begin{aligned}
\phi_{2}^{-1}\left(y_{2}\right) & =\left\{x \in X: y_{2} \in \phi_{2}(x)\right\} \\
& =\left\{x \in W_{2}: y_{2} \in \phi_{2}(x)\right\} \cup\left\{x \in X \backslash W_{2}: y_{2} \in \phi_{2}(x)\right\} \\
& =\left[W_{2} \cap\left(A_{2} \cap P_{2}\right)^{-1}\left(y_{i}\right)\right] \cup\left[\left(X \backslash W_{2}\right) \cap A_{i}^{-1}\left(y_{2}\right)\right] \\
& =A_{2}^{-1}\left(y_{i}\right) \cap\left[P_{2}^{-1}\left(y_{2}\right) \cap\left(\left(X \backslash W_{2}\right) \cap A_{2}^{-1}\left(y_{2}\right)\right)\right] \\
& =\left[A_{2}^{-1}\left(y_{2}\right) \cap P_{\imath}^{-1}\left(y_{2}\right)\right] \cup\left[\left(X \backslash W_{\imath}\right) \cap A_{\imath}^{-1}\left(y_{2}\right)\right]
\end{aligned}
$$

is non-empty open in $X$.

Finally, we define $\Psi: X \rightarrow 2^{X}$, by

$$
\Psi(x):=\Pi_{\imath \in I} \psi_{2}(x) \text { for each } x \in X,
$$

where

$$
\psi_{2}(x)= \begin{cases}\phi_{2}(x), & \text { if } i \in I_{0} \\ A_{2}(x), & \text { if } i \notin I_{0}\end{cases}
$$

Then each $\Psi(x)$ is a non-empty closed subset of $X$ and $\Psi$ is upper semicontinuous; so that by the Lemma again, $\Psi$ is a closed multimap. If we let $\widehat{y}=\left(y_{1}, \cdots, y_{n}\right) \in X$, then $\Psi^{-1}(\widehat{y})$ is a non-empty open subset of $X$. Therefore, by applying Theorem 1 to $\Psi$, we have that $\widehat{y} \in \Psi(\widehat{y})$, i.e. for each $i \in I$, $y_{\imath} \in \psi_{1}(\widehat{y})$. For each $i \in I_{0}, y_{\imath} \in \psi_{2}(\widehat{y})=\phi_{2}(\widehat{y})$. If $\widehat{y} \in W_{2}$, then

$$
y_{2} \in \psi_{2}(\widehat{y})=\left(A_{2} \cap P_{\imath}\right)(\widehat{y}) \subset P_{2}(\widehat{y}),
$$

which is a contradiction. Therefore, for each $i \in I_{0}, \widehat{y} \notin W_{2}$, i.e. $y_{2} \in \psi_{2}(\widehat{y})=A_{\imath}(\widehat{y})$ and $\left(A_{2} \cap P_{\imath}\right)(\widehat{y})=\emptyset$. Next, in case $i \notin I_{0}$, then $W_{\imath}=\emptyset$ and $\psi_{\imath}=A_{2}$. Therefore, $\widehat{y}_{\imath} \in \psi_{\imath}(\widehat{y})=A_{\imath}(\widehat{y})$ and $P_{\imath}(\widehat{y}) \cap A_{\imath}(\widehat{y})=\emptyset$. This completes the proof.

REMARKS. (i) In assumption (5), $W_{2}$ must be a proper subset of $X$. In fact, if $W_{2}=X$, then by applying Theorem 1 to the correspondence $\Pi_{\imath \in I}\left(A_{i} \cap P_{2}\right)$, we can get a fixed point $\widehat{x} \in \Pi_{\imath \in I}\left(A_{\imath} \cap P_{\imath}\right)(\widehat{x}) \subset \Pi_{\imath \in I} P_{\imath}(\widehat{x})$, which contradicts assumption (6).

(ii) Theorem 4 is quite different from the previous many equilibrium existence theorems (e g. $[3,4,5])$. In fact, in Theorem 4, we do not need the compact and convex assumption of the choice set $X_{2}$, but we only need the connectedness assumption. Also we do not need the convexity assumptions on the values $A_{\imath}(x)$ and $P_{i}(x)$ and strong open lower section assumptions; but we need the weaker open lower section property at some special point.

ACKNOWLEDGEMENT. The authors would like to thank Dr Cubiotti for his helpful comment which is the motivation of this paper, and this work was partially supported by KOSEF in 1995-1996.

\section{REFERENCES}

[1] BROWDER, F.E., The fixed point theory of multi-valued mappings in topological vector spaces, Math. Ann. 177 (1968), 283-301.

[2] FAN, K., Extensions of two fixed point theorems of F. E. Browder, Math. Z. 112 (1969), 234-240.

[3] MEHTA, G. and TARAFDAR, E., Infinite dimensional Gale-Nikaido-Debreu theorem and a fixed point theorem of Tarafdar, J. Econom. Theory 41 (1987), 333-339.

[4] TAN, K.-K. and YUAN, X.-Z., Maximal elements and equilibria for $\mathcal{U}$-majorized preferences, Bull. Austral. Math. Soc. 49 (1994), 47-54.

[5] TARAFDAR, E., A fixed point theorem and equilibrium point of an abstract economy, J. Math. Econom. 20 (1991), 211-218.

[6] DUGUNDJ, J. and GRANAS, A., Fixed Point Theory, Vol. 1, PWN, Warsaw (1982).

[7] AUBIN, J.P. and CELLINA, A., Differential Inclusion, Springer-Verlag, Berlin (1984). 


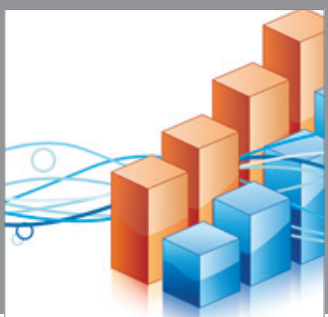

Advances in

Operations Research

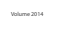

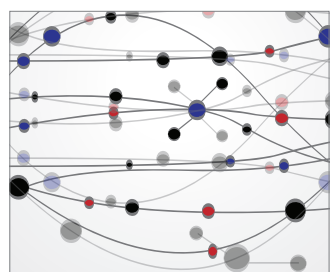

\section{The Scientific} World Journal
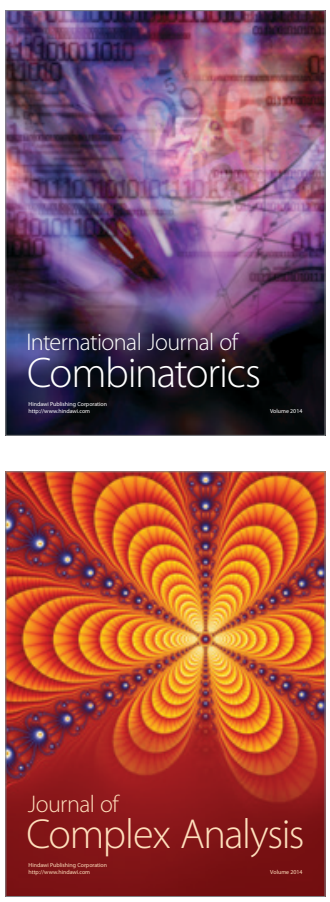

International Journal of

Mathematics and

Mathematical

Sciences
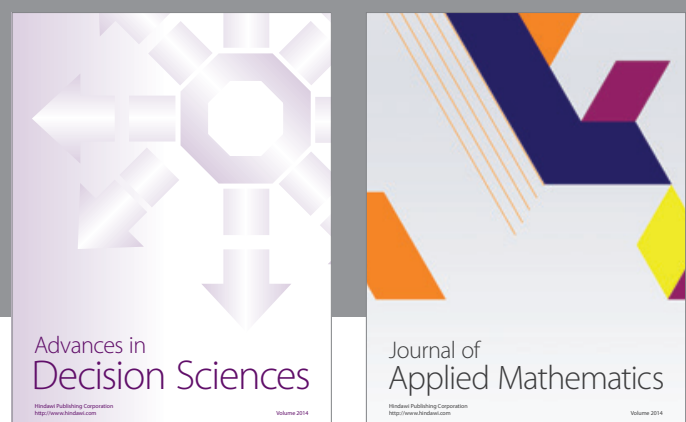

Journal of

Applied Mathematics
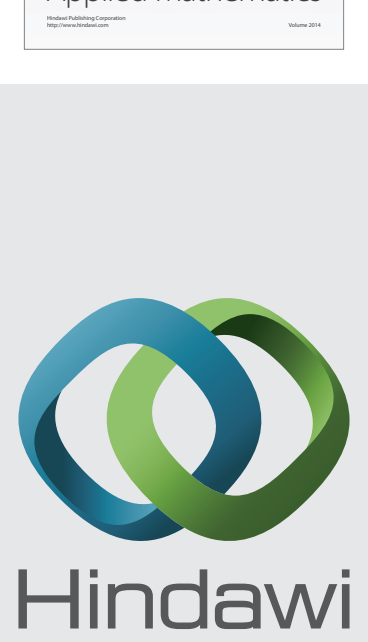

Submit your manuscripts at http://www.hindawi.com
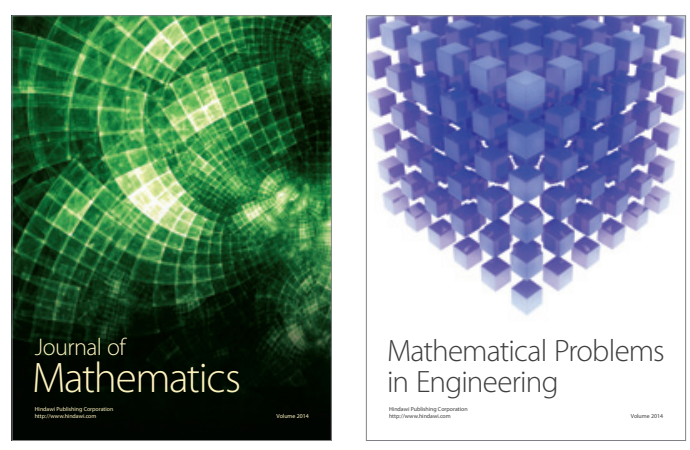

Mathematical Problems in Engineering
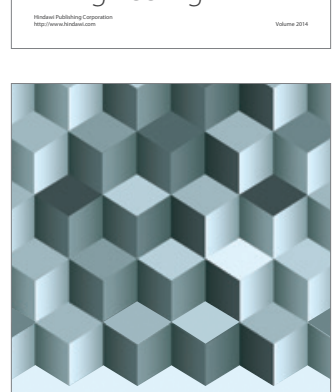

Journal of

Function Spaces
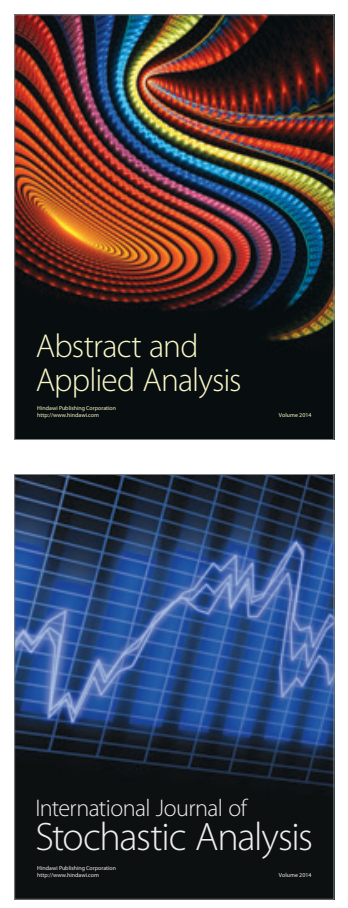

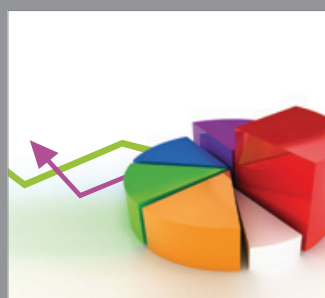

ournal of

Probability and Statistics

Promensencen
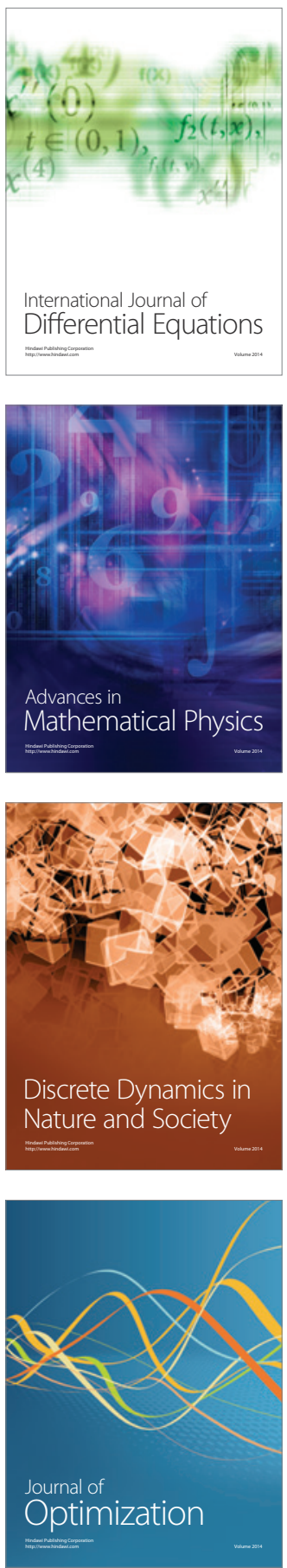